\title{
FURTHER TWO-DIMENSIONAL EFFECTS OF CYLINDERS ROLLING ON AN ELASTIC HALF-SPACE*
}

\author{
BY \\ A. M. ROBERTS \\ Monash University, Victoria, Australia.
}

1. Introduction. It has been shown by Galin [3] and independently by Sneddon [10] and Radok [7] that mixed boundary-value problems in two-dimensional dynamic elasticity for a half-space can be solved by suitably modifying the procedures of Mushelšvili [6]. In this context, three further types of mixed boundary-value problems occur, all related to the effect of a cylindrical stamp rolling with constant speed over the surface of a half space.

The first of these is the effect of an inflated "tire" which exerts a constant pressure over the contact region, which region is instantaneously at rest. We obtain expressions for the shear stress and the derivatives of the displacements over the contact. The surface shear is found to be infinite near the ends of the contact region, where it is postulated that slipping will occur. The width of the region of slip is calculated for various values of the speed of the tire and of the coefficient of friction between the tire and the half-space. If an appreciable resultant shear force is present, the boundary conditions are modified to allow for slipping over part of the contact region. The width of this region, where we assume limiting friction, is tabulated as a function of the above variables and of the resultant shear force.

Finally we solve the problem of a rolling rigid wheel, which, although less useful in the engineering sense, also demonstrates the general result that zones of high stress which occur at the ends of the contact region in all the problems discussed in this paper increase in width as the load speed approaches the speed of Rayleigh waves over the half space.

2. Equations of motion. For a load moving with a constant speed $V$ in the positive $x$ direction over the surface $y=0$ of the half-space $y<0$, it is appropriate to take a coordinate system moving with the load,

$$
x^{\prime}=x-V t, \quad y^{\prime}=y .
$$

If we introduce the dilatational and rotational wave speeds

$$
c_{1}^{2}=(\lambda+2 \mu) / \rho, \quad c_{2}^{2}=\mu / \rho,
$$

where $\lambda$ and $\mu$ are the Lamé constants and $\rho$ the density of the half-space, the potentials $\phi$ and $\psi$ defined by

$$
u=\partial \phi / \partial x^{\prime}+\partial \psi / \partial y^{\prime}, \quad v=\partial \phi / \partial y^{\prime}-\partial \psi / \partial x^{\prime},
$$

* Received December 17, 1969. 
where $(u, v)$ is the displacement, satisfy

$$
\nabla^{2} \phi=V^{2} / c_{1}^{2} \cdot \partial^{2} \phi / \partial x^{\prime 2}, \quad \nabla^{2} \psi=V^{2} / c_{2}^{2} \cdot \partial^{2} \psi / \partial x^{\prime 2},
$$

where

$$
\nabla^{2}=\partial^{2} / \partial x^{2}+\partial^{2} / \partial y^{\prime 2}
$$

For $V<c_{2}\left(<c_{1}\right)$, Eqs. (4) are elliptic with solutions

$$
\phi\left(x^{\prime}, y^{\prime}\right)=\operatorname{Re} f\left(z_{1}\right), \quad \psi\left(x^{\prime}, y^{\prime}\right)=\operatorname{Re} g\left(z_{2}\right),
$$

where

$$
z_{1,2}=x^{\prime}+i \beta_{1,2} y^{\prime}, \quad \beta_{1,2}=\left(1-V^{2} / c_{1,2}^{2}\right)^{1 / 2},
$$

and the complex functions $f$ and $g$ are to be analytic in $y^{\prime}<0$. It follows from (3) and (5) and the stress displacement relations that

$$
\begin{aligned}
v\left(x^{\prime}, y^{\prime}\right)= & -\beta_{1} \operatorname{Im} f^{\prime}\left(z_{1}\right)-\operatorname{Re} g^{\prime}\left(z_{2}\right), \quad u\left(x^{\prime}, y^{\prime}\right)=\operatorname{Re} f^{\prime}\left(z_{1}\right)-\beta_{2} \operatorname{Im} g^{\prime}\left(z_{2}\right) ; \\
& 1 / \mu \cdot \tau_{x x}=\left[2+c^{2}\left(m^{2}-2\right)\right] \cdot \operatorname{Re} f^{\prime \prime}\left(z_{1}\right)-2 \beta_{2} \cdot \operatorname{Im} g^{\prime \prime}\left(z_{2}\right), \\
& 1 / \mu \cdot \tau_{x y}=-2 \beta_{1} \cdot \operatorname{Im} f^{\prime \prime}\left(z_{1}\right)-\left(2-m^{2} c^{2}\right) \cdot \operatorname{Re} g^{\prime \prime}\left(z_{2}\right), \\
& 1 / \mu \cdot \tau_{y y}=-\left(2-m^{2} c^{2}\right) \cdot \operatorname{Re} f^{\prime \prime}\left(z_{1}\right)+2 \beta_{2} \cdot \operatorname{Im} g^{\prime \prime}\left(z_{2}\right),
\end{aligned}
$$

where primes on $f$ and $g$ indicate derivatives, and $c=V / c_{1}, m=c_{1} / c_{2}$. The abbreviations

$$
\begin{aligned}
K & =2-m^{2} c^{2}, \quad G_{1,2}=K \mp 2\left(\beta_{1} \beta_{2}\right)^{1 / 2}, \\
N(V) & =G_{1} G_{2}=K^{2}-4 \beta_{1} \beta_{2}, \quad b_{1,2}=\beta_{1,2}(2-K) /\left(K-2 \beta_{1} \beta_{2}\right),
\end{aligned}
$$

where both $b_{1}$ and $b_{2}$ are positive, will be used subsequently. The function $N(V)$ is the Rayleigh wave expression, which has a simple zero when $V=V_{R}$, the Rayleigh wave speed of the half-space. Expression $G_{1}$, and hence $N(V)$, is negative for $0<V<V_{B}$, and positive for $V_{R}<V<c_{2}$.

3. Inflated tire problem. An inflated tire rolling on a half-space will exert uniform pressure on the region of contact, and be instantaneously at rest in this region. We thus require that

$$
\begin{array}{lll}
\tau_{y y}\left(x^{\prime}, 0\right)=0, & \tau_{x y}\left(x^{\prime}, 0\right)=0, & \left|x^{\prime}\right|>a, \\
\tau_{y y}\left(x^{\prime}, 0\right)=-P, & \frac{\partial u}{\partial t}\left(x^{\prime}, 0\right)=0, & \left|x^{\prime}\right|<a,
\end{array}
$$

where the symmetry of the problem allows us to assume a symmetrical contact region. The value of $a$ is assumed to be determined beforehand, depending on the inflation of the tire and the weight of the vehicle.

It is convenient to obtain a solution in terms of the functions

$$
F_{1}(z)=-K \cdot f^{\prime \prime}(z)-2 i \beta_{2} \cdot g^{\prime \prime}(z), \quad F_{2}(z)=2 i \beta_{1} \cdot f^{\prime \prime}(z)-K \cdot g^{\prime \prime}(z),
$$

which have real parts proportional to the surface stresses as $\operatorname{Im} z \rightarrow 0-$. We may obtain $f$ and $g$ from the $F$ 's by inverting (11):

$f^{\prime \prime}(z)=1 / N(V) \cdot\left[-K \cdot F_{1}(z)+2 i \beta_{2} \cdot F_{2}(z)\right], \quad g^{\prime \prime}(z)=1 / N(V) \cdot\left[K \cdot F_{2}(z)+2 i \beta_{1} \cdot F_{1}(z)\right]$. 
Boundary conditions (10) may now be written

$$
\begin{array}{lll}
\operatorname{Re} F_{1}^{-}(t)=0, & \operatorname{Re} F_{2}^{-}(t)=0, & |t|>a, \\
\operatorname{Re} F_{1}^{-}(t)=-P / \mu, & \operatorname{Im} F_{2}^{r}(t)=P /\left(\mu b_{2}\right), & |t|<a,
\end{array}
$$

where $F^{ \pm}(t)=\lim _{y \rightarrow 0+} F(t \pm i y)$, and we have used $\partial / \partial t=-V \cdot \partial / \partial x^{\prime}$. The last of conditions (13) follows from the displacement expression (7), relations (12), and the second from last condition of (13). Conditions (13) specify separate Riemann problems for the analytic functions $F_{1}$ and $F_{2}$. The direct solution of Riemann problems of complex variable theory is developed by Galin [3], and summarized by this writer in the notation of the present paper in his thesis [8]. It is easily verified that

$$
\begin{aligned}
& F_{1}(z)=P /(\mu \pi i) \cdot \log [(z-a) /(z+a)], \\
& F_{2}(z)=-P /\left(\mu i b_{2}\right)+P /\left(\mu i b_{2}\right) \cdot\left(z-c_{0}\right) /\left(z^{2}-a^{2}\right)^{1 / 2},
\end{aligned}
$$

where $c_{0}$ is a real constant, and the branch cuts of the logarithm and surd functions are taken as the real axis from $-a$ to $a$, the branches chosen being those which have positive real values at plus infinity, satisfy (13).

It is now possible to evaluate any of the physical variables using (7), (8), (12) and (14). The quantities of greatest interest are the surface stress and displacement. The surface shear stress is given by

$$
\begin{aligned}
\tau_{x y}\left(x^{\prime}, 0\right) & =\mu \operatorname{Re} F_{2}^{-}\left(x^{\prime}\right) \\
& =P / b_{2} \cdot\left(x^{\prime}-c_{0}\right) /\left(a^{2}-x^{\prime 2}\right)^{1 / 2}, \quad\left|x^{\prime}\right|<a .
\end{aligned}
$$

If the resultant horizontal force exerted by the wheel is $T$, integration of (15) from $-a$ to $a$ gives

$$
c_{0}=-T b_{2} /(P \pi) .
$$

The vertical surface displacement is found to be

$$
\begin{aligned}
\left(\partial v / \partial x^{\prime}\right)\left(x^{\prime}, 0\right)= & \left(K-2 \beta_{1} \beta_{2}\right) / N(V) \cdot \operatorname{Re}\left[i b_{1} F_{1}^{-}\left(x^{\prime}\right)+F_{2}^{-}\left(x^{\prime}\right)\right] \\
= & P / \mu \cdot\left(K-2 \beta_{1} \beta_{2}\right) / N(V) \cdot\left\{1 / b_{2} \cdot\left(x^{\prime}-c_{0}\right) /\left(a^{2}-x^{\prime 2}\right)^{1 / 2}\right. \\
& \left.-b_{1} / \pi \cdot \log \left|\left(a-x^{\prime}\right) /\left(x^{\prime}+a\right)\right|\right\}, \quad\left|x^{\prime}\right|<a, \\
= & P b_{1} /(\mu \pi) \cdot\left(K-2 \beta_{1} \beta_{2}\right) / N(V) \cdot \log \left|\left(x^{\prime}-a\right) /\left(x^{\prime}+a\right)\right|, \quad\left|x^{\prime}\right|>a .
\end{aligned}
$$

Outside the loaded region, $\partial v / \partial x^{\prime}$ is positive if $x^{\prime}>a$, and negative if $x^{\prime}<-a$ for $V<V_{\boldsymbol{B}}$. This means that the load depresses the surface of the half space, as would be expected from the sign of $\tau_{y y}\left(x^{\prime}, 0\right)$. The horizontal surface displacement in $\left|x^{\prime}\right|>a$ is given by

$$
\begin{aligned}
\left(\partial u / \partial x^{\prime}\right)\left(x^{\prime}, 0\right) & =\left(K-2 \beta_{1} \beta_{2}\right) / N(V) \cdot \operatorname{Re}\left[-F_{1}^{-}\left(x^{\prime}\right)+i b_{2} F_{2}^{-}\left(x^{\prime}\right)\right] \\
& =-P / \mu \cdot\left(K-2 \beta_{1} \beta_{2}\right) / N(V) \cdot\left[1-\left(x^{\prime}-c_{0}\right) /\left(x^{\prime 2}-a^{2}\right)^{1 / 2}\right] .
\end{aligned}
$$

4. Discussion. (i) Displacement. It is apparent from (17) and (18) that the displacements become infinite as $V$ approaches $V_{R}$. This singularity has been noted by Craggs and Roberts [2], and further explained by Gol'dštein [4], who investigated the displacements and wavefronts for the transient problem of a Heaviside function load applied at the origin at $t=0$, and then moving with constant speed $V$. The above 
analysis shows that in the present problem, for $V<V_{R}$, a downward force on the halfspace depresses the surface, but for $V_{R}<V<c_{2}$, the sign of the displacement $v$ is reversed. It has been shown by Roberts [8], using the method of Cole and Huth [1], that this reversal occurs for any boundary-value problem involving a downward resultant force, in the speed range $V_{R}<V<c_{1}$ (a result which agrees with Gol'dštein's work on the transient problem). For $V>c_{1}$, no such anomaly is observed, the equations of motion (4) being hyperbolic, so that disturbances are carried backwards from the loaded region along the characteristics of these equations.

Both the transient and steady-state problems of a moving point load have been solved by Lansing [5], who obtains no anomalous surface displacements, so it appears that the present difficulties are peculiar to the two-dimensional situation. The advantage of two-dimensional steady-state investigations, however, is that mixed boundary-value problems may be solved, giving stress distributions over the contact region which may be of practical use, at least for load speeds below the Rayleigh wave speed.

(ii) Stress. We have from (16) that the points where the shear stress (15) and the slope of the displacement (17) change sign depend on the resultant horizontal force $T$. The fact that the shear stress takes both signs beneath the load means that frictions beneath a tire will act in both directions, the proportion in either direction depending on the sign and magnitude of $T$.

At the ends of the contact zone, the shear stress is infinite, behaving like $\left(a-x^{\prime}\right)^{-1 / 2}$. The physical explanation of this is that we do not permit the tire to push the surface horizontally apart (the horizontal displacement being fixed), so the shear stress buildup cannot be released (cf. Roberts [9]). This may be allowed for by deformation and slipping of the tire near $x^{\prime}= \pm a$, so we consider that the displacement boundary condition (10) will apply only to the half-space, not to the tire. The expressions for both surface displacements are integrable at $\pm a$, so that the displacement will be finite there, but the presence of infinite stress shows that linear elasticity theory is invalid in the neighborhood of these points.

We can estimate the width of the region where there is no slip by requiring the magnitude of the shear stress to be less than the coefficient of friction $(\Lambda)$ times the normal stress $(P)$. Application of this condition to (15) leads to

$$
\left(1+\Lambda^{2} b_{2}^{2}\right) \cdot x^{\prime 2}-2 c_{0} x^{\prime}+c_{0}^{2}-a^{2} \Lambda^{2} b_{2}^{2} \leq 0 .
$$

It is convenient to discuss this inequality in terms of the three variables $V, \Lambda$ and $c_{0}$ (or $T$ ) by first taking $c_{0}=0$ (i.e., zero resultant horizontal force). In this case, inequality (19) reduces to

$$
\left|x^{\prime} / a\right| \leq \Lambda b_{2}\left(1+\Lambda^{2} b_{2}^{2}\right)^{-1 / 2} .
$$

Values of $\left|x^{\prime} / a\right|$ satisfying the equality (20) are given in Table 1 for selected values of $\Lambda$ and $V$ for the case where $\lambda=\mu$.

It is apparent from Table 1 that for $\Lambda \geq 5$ there is no slip over virtually the whole contact zone for any value of $V<V_{R}$. For smaller values of $\Lambda$, the width of the zone of slipping increases as $\Lambda$ decreases and as $V$ increases. The widening of a zone where linear elasticity fails on increasing the load speed is found to be a standard phenomenon for the three mixed boundary-value problems discussed in this paper. It is not present in Galin's solutions [3] of the dynamic cases of the "first fundamental problem" ( $\tau_{x y}$ and $\tau_{y y}$ given on $\left.y^{\prime}=0\right)$, or the "second and third mixed problems" $\left(v\left(x^{\prime}, 0\right)\right.$ given with either 
TABLE 1.

Values of $\left|x^{\prime} / a\right|$ giving the width of the slip-free contact region from (20) for $c_{0}=0, \lambda=\mu$ and selected values of $\Lambda$ and $V$.

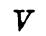

\begin{tabular}{lccccccc}
\hline & 0 & $.3 c_{2}$ & $.5 c_{2}$ & $\frac{2}{3} . c_{2}$ & $.8 c_{2}$ & $V_{R}=.92 c_{2}$ & $c_{2}$ \\
5 & 1 & 1 & 1 & .99 & .99 & .96 & 0 \\
1 & .95 & .94 & .92 & .88 & .79 & .56 & 0 \\
$\frac{1}{2}$ & .84 & .81 & .76 & .68 & .54 & .32 & 0 \\
$\frac{1}{3}$ & .71 & .68 & .61 & .52 & .40 & .22 & 0
\end{tabular}

$\tau_{x y}\left(x^{\prime}, 0\right)=0$ or $\tau_{x y}\left(x^{\prime}, 0\right)=\Lambda \tau_{y y}\left(x^{\prime}, 0\right)$ in $\left.-b<x^{\prime}<a\right)$, the nomenclature being Muskhelishvili's.

The presence of a driving force $T$ means that (19) has the solution

$$
\begin{aligned}
c_{0}-\Lambda b_{2}\left[a^{2}\left(1+\Lambda^{2} b_{2}^{2}\right)-c_{0}^{2}\right]^{1 / 2} & <x^{\prime}\left(1+\Lambda^{2} b_{2}^{2}\right) \\
& <c_{0}+\Lambda b_{2}\left[a^{2}\left(1+\Lambda^{2} b_{2}^{2}\right)-c_{0}^{2}\right]^{1 / 2},
\end{aligned}
$$

which zone is now centered on $x^{\prime}=c_{0} /\left(1+\Lambda^{2} b_{2}^{2}\right)$, which is negative for positive $T$. This asymmetry will widen the zone of slipping at one end of the contact region but reduce it at the other end, as illustrated in Table 2. The presence of a significant zone

TABLE 2.

Values of $x^{\prime} / a$ at the ends of the slip-free contact region from (21) for $T /(P a)=1, \Delta=1, \lambda=\mu$ for various values of $V$.
$\boldsymbol{V}$
$\boldsymbol{x}$
$-1,8$
$.3 c_{2}$
$-1,80$
$.5 c_{2}$
$\frac{2}{3} . c_{2}$
$.8 c_{2}$
$\Delta=1 \quad-1, .81 \quad-1, .80 \quad-.99, .76$
$-.98, .72$
$-.92, .61$

of slipping invalidates our boundary condition, which assumes an instantaneously still contact.

In summary, the presence of an applied shear force increases the tendency of a tire to slip. This slipping occurs at the front of the contact for acceleration, and at the back for deceleration. The present analysis remains valid for small $c_{0}$ (i.e., for $|T /(P a)| \ll \pi / b_{2}$ ), small $V$ and moderate to large $\Lambda(\Lambda>1)$, but otherwise the boundary conditions of the next section must be used.

5. Modified inflated tire problem. The above discussion suggests that for indentation by a tire which exerts a driving (or braking) force, the appropriate boundary-value problem would be

$$
\begin{aligned}
& \tau_{y y}\left(x^{\prime}, 0\right)=0, \quad \tau_{x y}\left(x^{\prime}, 0\right)=0, \quad\left|x^{\prime}\right|>a, \\
& \tau_{y y}\left(x^{\prime}, 0\right)=-P, \quad(\partial u / \partial t)\left(x^{\prime}, 0\right)=0, \quad-a<x^{\prime}<l, \\
& \tau_{y y}\left(x^{\prime}, 0\right)=-P, \quad \tau_{x y}\left(x^{\prime} ; 0\right)=\Lambda P, \quad l<x^{\prime}<a .
\end{aligned}
$$

This allows for slipping at the front of the contact zone (for a retarding force, the zone of slip would be at the rear of the contact), without permitting infinite shear stress near $x^{\prime}=a$. The additional parameter $l$ will be determined as a function of the load speed, the resultant shear, and the coefficient of friction.

The boundary conditions on $\tau_{v v}$ are the same as in the previous problem, so that $F_{1}(z)$ 
has the form given in (14). The remaining boundary conditions of (22) can be expressed in terms of $F_{2}(z)$ as

$$
\begin{aligned}
& \operatorname{Re} F_{2}^{-}(t)=0, \quad|t|>a, \\
& \operatorname{Im}{F_{2}^{-}}_{2}^{-}(t)=P /\left(\mu b_{2}\right), \quad-a<t<l, \\
& \operatorname{Re} F_{2}^{-}(t)=\Lambda P / \mu, \quad l<t<a .
\end{aligned}
$$

This Riemann problem for $F_{2}$ may be solved by multiplying through by the real factor $|(t+a)(t-l)|^{1 / 2}$. We obtain from (23)

$$
\begin{array}{rlrl}
\operatorname{Re}\left[(t+a)^{1 / 2}(t-l)^{1 / 2} \cdot F_{2}(t)\right]^{-} & =0 \quad|t|>a, \\
& =P /\left(\mu b_{2}\right) \cdot(t+a)^{1 / 2}(l-t)^{1 / 2} & -a<t<l, \\
& =\Lambda P / \mu \cdot(t+a)^{1 / 2}(t-l)^{1 / 2} \quad l<t<a,
\end{array}
$$

where the branch cut of the surd in the complex plane lies along the real axis from $-a$ to $l$, and the branch is that which is positive real at plus infinity. The function which is analytic in the lower half of the complex plane and satisfies (24) is given by the Cauchy integral

$(z+a)^{1 / 2}(z-l)^{1 / 2} F_{2}(z)=-1 /(\pi i) \cdot \int_{-\infty}^{\infty} \operatorname{Re}\left[(t+a)^{1 / 2}(t-l)^{1 / 2} \cdot F_{2}(t)\right]^{-} /(t-z) \cdot d t$.

Substituting (24) into (25) and performing the integration leads to

$$
\begin{aligned}
& F_{2}(z)=\Lambda P i /(\mu \pi)\left\{\log W(z)+\left(2 a^{2}-2 a l\right)^{1 / 2}(z+a)^{-1 / 2}(z-l)^{-1 / 2}\right. \\
& \left.+\frac{1}{2}(2 z+a-l)(z+a)^{-1 / 2}(z-l)^{-1 / 2} \log \left[\left[3 a-l+\left(8 a^{2}-8 a l\right)^{1 / 2}\right] /(a+l)\right]\right\} \\
& +P i /\left(\mu b_{2}\right)\left[1-\frac{1}{2}(2 z+a-l)(z+a)^{-1 / 2}(z-l)^{-1 / 2}\right] \\
& +i P c_{0} /\left(\mu b_{2}\right) \cdot(z+a)^{-1 / 2}(z-l)^{-1 / 2}
\end{aligned}
$$

where

$$
\begin{aligned}
W(z)=\left[(z-l)^{1 / 2}-(z+a)^{1 / 2}\right]\left[a-z+\left(2 a^{2}-2 a l\right)^{1 / 2}+(z+a)^{1 / 2}(z-l)^{1 / 2}\right]^{-1} \\
\cdot\left[(z-l)^{1 / 2}+(z+a)^{1 / 2}\right]^{-1}\left[a-z+\left(2 a^{2}-2 a l\right)^{1 / 2}-(z+a)^{1 / 2}(z-l)^{1 / 2}\right] .
\end{aligned}
$$

In this expression, the square roots denote those branches which are positive real at plus infinity, and the branch cuts extend from the branch points along the real axis to minus infinity. This definition agrees with that given previously for the product $(z+a)^{1 / 2}(z-l)^{1 / 2}$. The branch of the logarithm is that which is real and positive at plus infinity.

The terms containing $\Lambda P$ in expression (26) come from the last part of the boundary conditions (22), while those involving $P$ only come from the second condition of (22). It follows from the nature of Cauchy integrals that both these terms will be of order $z^{-2}$ at infinity. We require $F_{2}$ (and hence the stresses) to be of order $z^{-1}$ at infinity, and so add the term containing $c_{0}$. It can be shown by writing $F_{2}(z)$ as a Cauchy integral and expanding in powers of $z^{-1}$ that this coefficient is related to the resultant shear force $\left[8\right.$, p. 56], and we have chosen the constants multiplying $c_{0}$ in (26) so that expression (16) relating $c_{0}$ to the resultant shear is still valid.

Since $\tau_{x y}$ is related to the real part of $F_{2}$, it is apparent from (26) that the shear stress will be infinite at $-a$ and $l$ due to the surds in the denominator. We select $l$ so that 
the coefficient of $(z+a)^{-1 / 2}(z-l)^{-1 / 2}$ in (26) vanishes at $z=l$. This leads to the equation $2(2-2 A)^{1 / 2} /(1+A)+\log \left[(3-A) /(1+A)+2(2-2 A)^{1 / 2} /(1+A)\right]$

$$
=\pi /\left(\Lambda b_{2}\right)+4 T /(2 a P) \cdot 1 /(1+A) \cdot 1 / \Lambda \text {, }
$$

where we have written

$$
A=l / a<1,
$$

and used expression (16) to put $c_{0}$ in terms of $T /(2 a P)$, which represents the ratio of the mean shear per unit length $\left(\frac{1}{2} T / a\right)$ to the pressure per unit length $(P)$ over the contact zone. The other parameters involved in Eq. (27), which is to be solved for $A$ (or $l$ ), are $\Lambda$ and $V$.

Before solving Eq. (27) explicitly, we note that we may use it to simplify expression (26) and hence obtain some information on the surface shear stress. Elimination of the logarithm with the constant argument from (26) leads to

$$
\begin{aligned}
F_{2}(z)= & \Lambda P i /(\mu \pi) \cdot \log W(z)+i P /\left(\mu b_{2}\right) \\
& -2 P i /\left(b_{2} \mu \pi\right) \cdot 1 /(a+l) \cdot(z-l)^{1 / 2}(z+a)^{-1 / 2}\left[\Lambda b_{2}\left(2 a^{2}-2 a l\right)^{1 / 2}+c_{0} \pi\right] .
\end{aligned}
$$

It must be remembered when using (29) that $l$ is now given by (27) as a function of $\Delta$, $V$ and $c_{0}$. The surface shear stress is given by

$$
\begin{aligned}
\tau_{x u}\left(x^{\prime}, 0\right) & =\Lambda P-2 \Lambda P / \pi \cdot \arg \left[\left(x^{\prime}+a\right)^{1 / 2}+i\left(l-x^{\prime}\right)^{1 / 2}\right] \\
& -2 \Lambda P / \pi \cdot \arg \left[a-x^{\prime}+\left(2 a^{2}-2 a l\right)^{1 / 2}+i\left(x^{\prime}+a\right)^{1 / 2}\left(l-x^{\prime}\right)^{1 / 2}\right] \\
& -2 P /\left(b_{2} \pi\right) \cdot 1 /(a+l) \cdot\left|\left(x^{\prime}-l\right) /\left(x^{\prime}+a\right)\right|^{1 / 2}\left[\Lambda b_{2}\left(2 a^{2}-2 a l\right)^{1 / 2}+c_{0} \pi\right],
\end{aligned}
$$

in $-a<x^{\prime}<l$. It is now apparent that the surface stress is continuous at $x^{\prime}=l$, and satisfies the boundary condition in $l<x^{\prime}<a$. It is still infinite at $x^{\prime}=-a$. The last term of (30) is of uniform sign in $-a<x^{\prime}<l$, while the remaining terms combine to be monotonic increasing from 0 at $-a$ to $\Lambda P$ at $l$. We find that the width of the region near $-a$ where the magnitude of the shear stress exceeds $\Lambda P$ is small in most practical cases. At this stage it is worth noting that the boundary value problem equivalent to (22) but with slip at both ends of the contact can be formally solved, but the algcbraic complication of the solution makes it difficult to extract any useful information, especially since the present solution keeps the zone of high shear narrow.

We now discuss the values of $A$ obtained from Eq. (27), which are given in Table 3.

\section{TABLE 3.}

Values of $A=l / a$ satisfying (27), and the dimensionless lower limit of linear elasticity $\left(x^{\prime} / a\right)$ in parentheses,

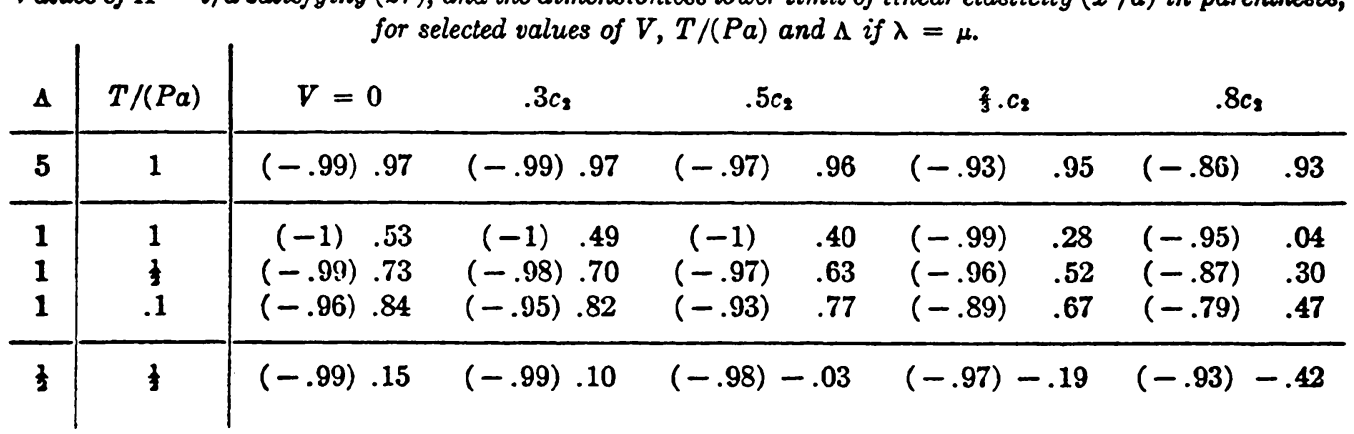


The figures in brackets are the points near $x^{\prime}=-a$ at which the shear stress equals $\Delta$ times the normal force (found approximately by equating the last term of expression $(30)$ to $\Lambda P$ ) in dimensionless form. Table 3 may thus be interpreted as giving the (dimensionless) width of the region where there is no slip, with linear elasticity being inapplicable for values of $x^{\prime} / a$ less than the figure in brackets, and limiting friction holding for $x^{\prime} / a$ greater than the other figure. The entries for $\Lambda=1, T /(P a)=1$ may be compared with Table 2.

It is seen that as $\Lambda$ gets smaller, the zone of slipping extends, as would be expected. If $\Lambda$ was sufficiently small, there would be slipping over the whole of the contact region, this being the "third mixed problem" considered by Galin [3]. The extension of the width of the zone of slip as the load speed increases is again apparent in Table 3 . It would be interesting if experimental verification of this widening could be obtained. The width of the region where the shear stress is large enough to give slipping near $x^{\prime}=-a$ is now pleasingly small $(<a / 10)$ in almost every case. It thus seems that the present boundary-value problem yields information on the distribution of stress beneath a tire moving with given speed and exerting a resultant shear (driving) force. For a freely rolling tire, the analysis given earlier can be used.

6. Rolling rigid wheel. The displacement conditions beneath a rolling rigid wheel may be obtained by reference to Fig. 1 . The instantaneous motion of $P\left(x^{\prime}, y^{\prime}\right)$ is per-

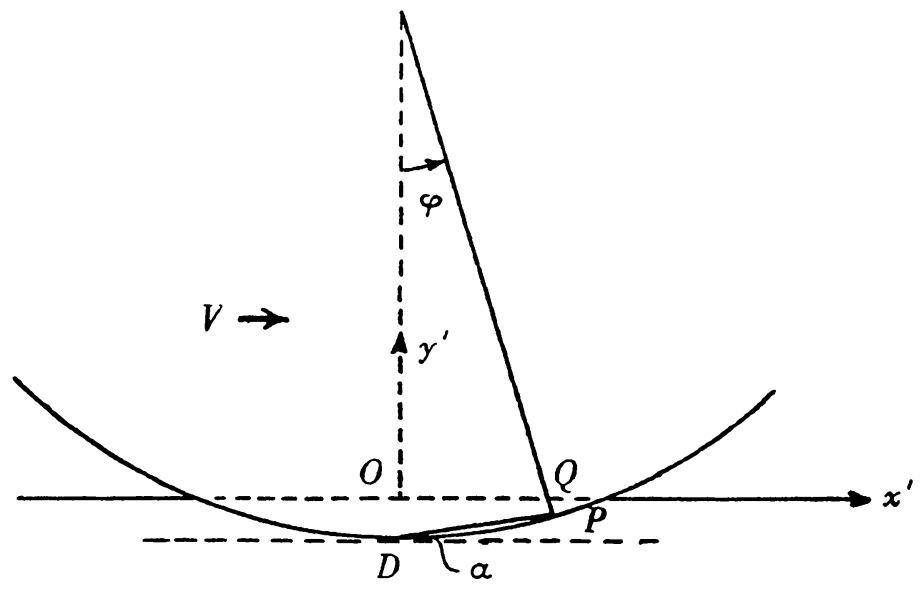

Frg. 1. Rolling wheel.

pendicular to $D P$, so that the components of velocity satisfy

$$
(\partial u / \partial t) /(\partial v / \partial t)=-\tan \alpha=-\left(y^{\prime}+d\right) / x^{\prime},
$$

where $d=O D$ is the depth of the indentation. The wheel (radius $R$ ) has equation

$$
x^{\prime 2}+\left[y^{\prime}-(R-d)\right]^{2}=R^{2},
$$

80

$$
\left(y^{\prime}+d\right) / x^{\prime}=\frac{1}{2} x^{\prime} / R+O\left(R^{-2}\right) .
$$

The vertical displacement of $Q\left(x^{\prime}, 0\right)$ to $P$ is 


$$
\begin{aligned}
v\left(x^{\prime}, 0\right) & =R-d-R \cos \varphi \\
& =(R-d)\left[1-R\left\{x^{\prime 2}+(R-d)^{2}\right\}^{-1 / 2}\right] \\
& =-d+(R+2 d){x^{\prime}}^{2} /\left(2 R^{2}\right)+O\left(R^{-3}\right) .
\end{aligned}
$$

It follows from (31), (32) and (33) that

$$
\begin{aligned}
& -1 / V \cdot(\partial v / \partial t)=\left(\partial v / \partial x^{\prime}\right)\left(x^{\prime}, 0\right)=(R+2 d) x^{\prime} / R^{2}=q\left(\beta_{1} / \beta_{2}\right)^{1 / 2} x^{\prime}, \\
& -1 / V \cdot(\partial u / \partial t)=\left(\partial u / \partial x^{\prime}\right)\left(x^{\prime}, 0\right)=-\frac{1}{2} x^{\prime 2}(R+2 d) / R^{3}=-r x^{\prime 2} .
\end{aligned}
$$

A double displacement boundary condition is thus applicable to the problem of a rolling rigid wheel.

We thus solve the steady state case of the "first mixed boundary value problem", namely

$$
\begin{aligned}
\tau_{y y}\left(x^{\prime}, 0\right) & =0, \quad \tau_{x y}\left(x^{\prime}, 0\right)=0, \quad x^{\prime}<-b, x^{\prime}>a, \\
\left(\partial u / \partial x^{\prime}\right)\left(x^{\prime}, 0\right) & =-r x^{\prime 2}, \quad\left(\partial v / \partial x^{\prime}\right)\left(x^{\prime}, 0\right)=q\left(\beta_{1} / \beta_{2}\right)^{1 / 2} x^{\prime}, \quad-b<x^{\prime}<a,
\end{aligned}
$$

where $a, b, q$ and $r$ are considered known. These boundary conditions may be expressed in terms of the complex functions $f$ and $g$ using (7) and (8). The functions $F_{1}$ and $F_{2}$ of formula (11) may then be introduced to give the Riemann problems

$$
\begin{aligned}
\operatorname{Re}\left[-F_{1}^{-}(t)+i b_{2} F_{2}^{-}(t)\right] & =N(V) /\left(K-2 \beta_{1} \beta_{2}\right) \cdot\left(-r t^{2}\right), \\
\operatorname{Re}\left[i b_{1} F_{1}^{-}(t)+F_{2}^{-}(t)\right] & =N(V) /\left(K-2 \beta_{1} \beta_{2}\right) \cdot q\left(\beta_{1} / \beta_{2}\right)^{1 / 2} t
\end{aligned}
$$

in $-b<t<a$, the real parts of the $F^{\prime} \mathrm{s}$ vanishing for other values of $t$. If we extend the definition of the $F$ 's into the upper half-plane by

$$
F(z)=-\bar{F}(z)=-\overline{F(\bar{z})} \text { for } \operatorname{Im} z>0,
$$

a bar indicating the complex conjugate, then it follows that the linear combinations

$$
K_{1,2}(z)=F_{1}(z) \pm i\left(\beta_{2} / \beta_{1}\right)^{1 / 2} F_{2}(z)
$$

satisfy the Hilbert problems

$$
\begin{aligned}
K_{2}^{+}(t)+M K_{2}^{-}(t) & =2 G_{2} /\left[1+\left(\beta_{1} \beta_{2}\right)^{1 / 2}\right] \cdot\left(-r t^{2}+i q t\right), \\
K_{1}^{+}(t)+1 / M \cdot K_{1}^{-}(t) & =2 G_{1} /\left[1+\left(\beta_{1} \beta_{2}\right)^{1 / 2}\right] \cdot\left(-r t^{2}-i q t\right)
\end{aligned}
$$

in $-b<t<a$, the $K$ 's being analytic across the rest of the real axis. The constant $M$ is defined by

$$
\begin{aligned}
M=-G_{2} / G_{1} \cdot\left[1-\left(\beta_{1} \beta_{2}\right)^{1 / 2}\right] /\left[1+\left(\beta_{1} \beta_{2}\right)^{1 / 2}\right] & \\
& \rightarrow\left(m^{2}+1\right) /\left(m^{2}-1\right)=\kappa \text { as } V \rightarrow 0,
\end{aligned}
$$

the constant $\alpha$ being used by Mushelišvili [6, Sec. 114], in solving the corresponding static problem. The second of (38) in the static case is the conjugate of the first.

The boundary-value problems (38) have the solutions

$$
\begin{aligned}
K_{2}(z)= & N(V) \cdot\left(\beta_{1} \beta_{2}\right)^{-1 / 2} \cdot\left(i q z-r z^{2}\right) /(K-2) \\
& -N(V) \cdot\left(\beta_{1} \beta_{2}\right)^{-1 / 2} \cdot P(z) /(K-2) \cdot(z+b)^{i \beta-1 / 2}(z-a)^{-i \beta-1 / 2}, \\
K_{1}(z)= & -K_{2}(z)
\end{aligned}
$$


where

$$
\begin{aligned}
\beta & =1 /(2 \pi) \cdot \log M, \\
P(z) & =-r z^{3}+(i q-r A) z^{2}+(i q A+r B) z+c_{0}^{*}-i q B-r C, \\
A & =-a\left(\frac{1}{2}+i \beta\right)+b\left(\frac{1}{2}-i \beta\right), \quad B=\frac{1}{2}(a+b)^{2}\left(\frac{1}{4}+\beta^{2}\right), \\
C & =\frac{1}{6} \cdot\left(\frac{1}{4}+\beta^{2}\right)(a+b)^{2}\left[-a\left(\frac{3}{2}-i \beta\right)+b\left(\frac{3}{2}+i \beta\right)\right],
\end{aligned}
$$

$c_{0}^{*}$ being a complex constant. Using the expansions of Cauchy integral expressions for the $F$ 's at large $|z|$, we find that

$$
c_{0}^{*}=(K-2) / N(V) \cdot\left(\beta_{1} \beta_{2}\right)^{1 / 2} /(\mu \pi) \cdot\left[\left(\beta_{2} / \beta_{1}\right)^{1 / 2} T+i P^{*}\right],
$$

where $\left(P^{*}, T\right)$ is the resultant force exerted by the wheel on the half space [8, p. 61]. The branch cut of the surds in (40) lies along the real axis between $-b$ and $a$, and we select that branch which is real positive at plus infinity. The formal solution of problem (35) is now complete. The most interesting physical quantities are the stresses on the contact zone, which will be important in wheel construction and wear.

The surface displacements for $x^{\prime}>a$ and $x^{\prime}<-b$ are

$$
\begin{aligned}
\left(\partial u / \partial x^{\prime}\right)\left(x^{\prime}, 0\right)= & -r x^{\prime 2} \\
& -\left(x^{\prime}-a\right)^{-1 / 2}\left(x^{\prime}+b\right)^{-1 / 2} \cdot \operatorname{Re}\left[P\left(x^{\prime}\right) \cdot\left(x^{\prime}+b\right)^{i \beta} \cdot\left(x^{\prime}-a\right)^{-i \gamma}\right], \\
\left(\partial v / \partial x^{\prime}\right)\left(x^{\prime}, 0\right)= & q x^{\prime}\left(\beta_{1} / \beta_{2}\right)^{1 / 2} \\
& -\left(x^{\prime}-a\right)^{-1 / 2}\left(x^{\prime}+b\right)^{-1 / 2} \cdot \operatorname{Im}\left[P\left(x^{\prime}\right) \cdot\left(x^{\prime}+b\right)^{i \beta} \cdot\left(x^{\prime}-a\right)^{-i \beta}\right] .
\end{aligned}
$$

The surface stresses in $-b<x^{\prime}<a$ are

$$
\begin{aligned}
\tau_{y y}\left(x^{\prime}, 0\right)= & \mu \operatorname{Re}{F_{1}}_{1}^{-}\left(x^{\prime}\right)=\frac{1}{2} \mu \operatorname{Re}\left[K_{1}^{-}\left(x^{\prime}\right)+K_{2}^{-}\left(x^{\prime}\right)\right] \\
= & \mu[-N(V)]^{1 / 2} \cdot\left(1-\beta_{1} \beta_{2}\right)^{-1 / 2} \cdot\left(a-x^{\prime}\right)^{-1 / 2}\left(x^{\prime}+b\right)^{-1 / 2} . \\
& \cdot \operatorname{Im}\left[\left(x^{\prime}+b\right)^{i \beta} \cdot\left(a-x^{\prime}\right)^{-i \beta} \cdot P\left(x^{\prime}\right)\right], \\
\tau_{x y}\left(x^{\prime}, 0\right)= & \operatorname{Re} F_{2}^{-}\left(x^{\prime}\right)=\frac{1}{2} \mu \operatorname{Re}\left[1 / i \cdot\left(\beta_{1} / \beta_{2}\right)^{1 / 2}\left\{K_{1}^{-}\left(x^{\prime}\right)-K_{2}^{-}\left(x^{\prime}\right)\right\}\right] \\
= & \mu[-N(V)]^{1 / 2} \cdot\left(1-\beta_{1} \beta_{2}\right)^{-1 / 2} \cdot\left(a-x^{\prime}\right)^{-1 / 2}\left(x^{\prime}+b\right)^{-1 / 2} \\
& \cdot \operatorname{Re}\left[\left(x^{\prime}+b\right)^{i \beta}\left(a-x^{\prime}\right)^{-i \beta} \cdot P\left(x^{\prime}\right)\right] .
\end{aligned}
$$

The presence of complex powers in these expressions leads to oscillation of the surface stresses near the ends of the contact zone, as well as magnification of their size due to the inverse square root terms. For the case $a=b, T=0$ and $\lambda=\mu$, the region where the normal stress has one (negative) sign is $\left|x^{\prime} / a\right|<.997$ for $V=.8 c_{2}$, this region occupying virtually all the contact region at lower load speeds, and decreasing to zero at the Rayleigh wave speed, where a steady state is not attainable. This is the third occurrence of widening of an anomalous stress region at high load speeds.

The lack of symmetry in the present problem means that the position of both ends of the contact region must be known. A further condition on our solution may be obtained by considering the moment $\mathfrak{T}$ exerted by the wheel on the half-space. We have

$$
\begin{aligned}
& \mathscr{T}=\int_{-b}^{a} \tau_{y y}(t, 0) \cdot t \cdot d t \\
&=-\left(\beta_{1} \beta_{2}\right)^{-1 / 2} \pi \mu N(V) /(K-2) \cdot\left\{\beta(a+b) \cdot \operatorname{Re} c_{0}^{*}+\frac{1}{2}(a-b) \cdot \operatorname{Im} c_{0}^{*}\right. \\
&\left.+\left(\frac{1}{4}+\beta^{2}\right)(a+b)^{2}(a-b) / 12 \cdot[2 r(a+b)+3 q]\right\} .
\end{aligned}
$$


If $\mathfrak{T}=0$; the solution of $(46)$ is symmetric:

$$
a=b, \quad T=0, \quad \Re=0 .
$$

If $T, P^{*}$, and $\mathfrak{T}$ are prescribed, then the values of $a$ and $b$ are connected through (46), and we only need know the total width of the contact region $a+b$ to find $a$ and $b$. In the symmetric case, it follows from the vanishing of the resultant shear force that $\tau_{x y}$ will take both signs over the contact, so that friction will act in both directions beneath a rigid wheel. For the inflated tire problem, it follows from (15) that the resultant moment is

$$
\Re=\frac{1}{2} a^{2} P \pi / b_{2},
$$

so that prescribing $\Re$ in that problem is equivalent to prescribing $a$. A similar but more involved result would follow from (30) for a tire with a zone of slip. It follows that the rolling rigid wheel problem will always contain one more parameter which must be determined by measurement than the inflated tire problems. Hence the latter type of problem provides more useful results.

We add a remark on the determination of the width of indentation from the forces applied to the indentor. The classical Hertz problem ("second mixed problem") of a vertical punch pressed into a smooth surface has a solution of which the width of the contact region is given in terms of the resultant force exerted (and elastic constants). Such a relation is obtained by requiring the stress to be finite at the ends of the (symmetrical) contact region in the same way as we obtained Eq. (27). Such a condition imposed on the rigid wheel problem gives the two complex conditions

$$
P(-b)=0=P(a),
$$

which over specify the problem. Eqs. (49) imply that $T=0, P^{*}$ is specified in terms of $q, r$ and $V$, and $a=b$, either being specified as a function of the same quantities of the order of $R$. The equivalent result for the Hertz problem gives $a$ of the order of $R^{1 / 2}$, which will not be terribly small. It appears that in our problem, the shape and ends of the contact region must be specified when the problem is posed, independently of the forces exerted by the wheel. The best we can do is relate $a$ and $b$ through (46).

Acknowledgment. The writer would like to thank Professor J. W. Craggs for bringing the above problems to his attention.

\section{REFERENCES}

[1] J. D. Cole and J. H. Huth, Stresses produced in a half plane by moving loads, J. Appl. Mech. 25, 433-436 (1958)

[2] J. W. Craggs and A. M. Roberts, On the motion of a heavy cylinder over the surface of an elastic half space, J. Appl. Mech. 34, 207-209 (1967)

[3] L. A. Galin, Contact problems of the theory of elasticity, GITTL, Moscow, (Russian), 1963

[4] R. V. Gol'dštein, Rayleigh waves and resonance phenomena in elastic bodies, J. Appl. Math. Mech. 29, 608-619 (1965)

[5] D. L. Lansing, The displacements in an elastic half space due to a moving concentrated normal load, NASA Technical Report R-238, 1966

[6] N. I. Mushelišili, Some fundamental problems in the mathematical theory of elasticity. Fundamental equations, plane theory of elasticity and bending, Izdat. Akad. Nauk SSSR, Moscow, 1949; English transl., Noordhoff, Groningen, 1953

[7] J. R. M. Radok, On the solution of problems of dynamic plane elasticity, Quart. Appl. Math. 14, 289-298 (1956) 
[8] A. M. Roberts, Two-dimensional effects of loads moving on the surface of elastic and thermoelastic hulf spaces, $\mathrm{Ph}$. D. Thesis, University of Melbourne, 1969

[9] A. M. Roberts, A two-dimensional mixed boundary-value problem in elasticity, Quart. Appl. Math. $28,445-449$ (1970)

[10] 1. N. Sneddon, The stress produced by a pulse of pressure moving along the surface of a semi-infinite solid, Rend. Circ. Mat. Palermo (2) 1, 57-62 (1952) 